\title{
Beamsteering for ultra-high data-rate optical wireless communications
}

\author{
Dominic C O’Brien',

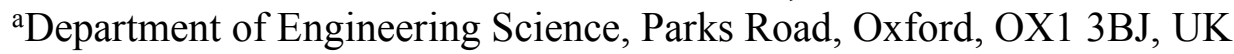

\begin{abstract}
Beamsteering systems offer the potential to extend the fibre-based telecommunications infrastructure with wireless point-to-point data links that are transparent to modulation scheme and data rate. In this paper we introduce beamsteering, and discuss the challenges in implementing and using such systems that use this approach in indoor environments. Results from a number of demonstration systems are presented, including ultra-high data rate indoor optical wireless, operating at up to $400 \mathrm{Gbit} / \mathrm{s}$.
\end{abstract}

Keywords: Beamsteering, Optical Wireless

\section{INTRODUCTION}

The telecommunications network is evolving into an all-optical core, using optical fibres, with wireless access to the network at the 'edges', wherever possible. This is leading to exponential growth in mobile wireless data traffic, with much of that traffic originating and terminating indoors. Provision of wireless capacity that can meet this demand is increasingly challenging due to the so-called spectrum 'crunch'. In the RF domain this is being addressed by the move to higher frequencies, such as the 30 and $60 \mathrm{GHz}$ bands, where there is unlicensed and/or available spectrum. In the optical domain there is a focus on LiFi to provide coverage density, and beamsteered optical communications to provide extremely high capacity with more limited coverage density.

Both high frequency RF and beamsteered optical communications provide a line-of-sight channel, and require the same basic elements. At the transmitter there must be a steering device that directs radiation from source to destination, and at the receiver there is usually a device that steers light from the transmitter into the system reception field of view. Together these steering devices work to connect transmitter to receiver (and vice-versa), but there must also be a tracking mechanism that allows the transmitter to locate the receiver (and vice-versa), and to control the steering devices to maintain a link. Finally, there must be some multiplexing method that allows point-to-multipoint transmission.

This paper focuses on optical beamsteering, but it should be noted that there are similar challenges for RF techniques in the mm-wave regions of the spectrum and beyond, where the propagation characteristics of the radiation are similar. In this paper we review techniques available for beamsteering and present results from a spatial light modulator based technique. (It should be noted that this is not intended to be a comprehensive review of all activities in this area, rather a brief overview of representative techniques).

\section{BEAMSTEERING TECHNIQUES}

The ideal beamsteering device would be solid-state, passive, with large aperture, wide steering angle and short response time. No devices currently meet all these goals simultaneously. Galvanometer type mirrors can provide wide steering angle and large aperture, but can be bulky and slow. There are designs such as [1] that are compact and can have relatively fast response, but these are not solid-state. Micro-Electrical-Mechanical-Systems (MEMs) mirrors are available commercially [2] and can provide high-speed steering through a narrow range of angles with limited aperture. In addition novel devices that allow wide angle and focusing exist[3]. In most cases there is a compromise between steering angle and aperture, and although optical systems can increase steering angle, conservation of etendue leads to a proportionate decrease in aperture. The product of the steering angle and aperture therefore serves a useful measure of the steering 'power' of a particular device.

Electrowetting devices[4] and liquid-filled prisms[5] have also been researched, and diffractive steering with Spatial Light Modulators has also been demonstrated by a number of groups[6-8]. In this approach wavefront control is achieved by control of the phase. Waveguide based beamsteerers that use arrays of waveguides with integrated phase shifters, outcouplers and in some cases sources have also been demonstrated[9-11], and similar concepts are being pursued in the context of LIDAR solid-state scanning systems. It is also feasible to use a fixed grating, or series of elements, and 
control the wavelength $[12,13]$. Such an element can be entirely passive, but the design of the element is usually constrained in some way, and achieving high steering angles can be challenging.

\section{BEAMSTEERING SYSTEMS AND DEMONSTRATIONS}

\subsection{System geometries}

Figure 1 shows a typical beamsteering system. Light from an optical fibre enters a transmitter, and is steered towards a receiver unit. Different types of receiver can be considered: The most challenging case is where the light is coupled back into an optical fibre, as the receiver must be pointed and aligned with the incoming beam. However, this offers the opportunity of bi-directional operation of the link, and the use of commodity fibre-based receiver components and standard fibre transmission protocols. In [14]mechanical alignment of the receiver is used to achieve the necessary alignment, whereas in $[8,15]$ two symmetrical SLM based systems are used.

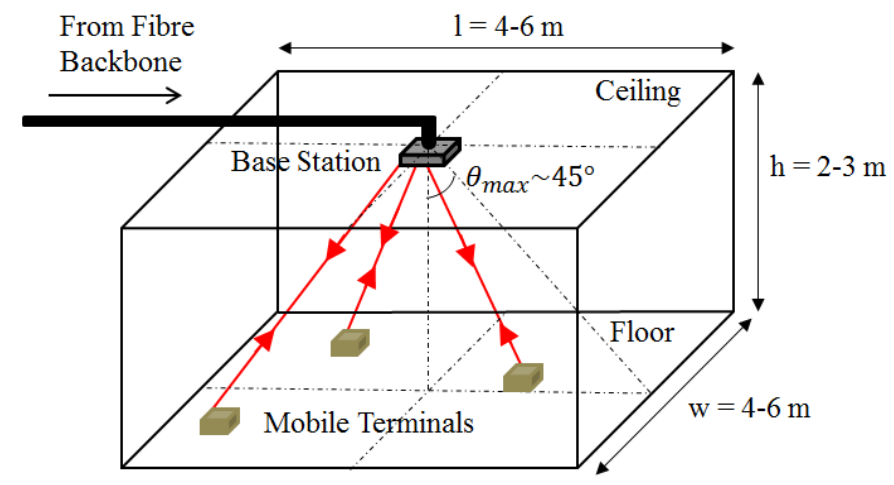

Figure 1. beamsteering system

\subsection{Tracking}

Beamsteering systems require tracking systems at the transmitter to locate the receiver and often at the receiver, in order to locate the transmitter. At the base station the tracking system must operate over wide field of view, and locate and track multiple terminals, whilst the receiver is often tracking a single incoming signal. 'Searchlight' techniques where the communications beam of light from the transmitter sweeps the coverage field of view are inefficient in such cases, as the receiver also needs to search in a similar way, leading to potentially long delays in signal acquisition. The use of beaconbased tracking with beacons on the terminals allows the base station to know the positions of the terminals, and such as system can be implemented using low-cost silicon cameras and LED beacons[16]. For the terminals a simpler tracker can be implemented by sensing the angle of arrival of the communications beam, either using a camera or a quad-detector. For less stringent requirements on alignment the use of WiFi and other indoor positioning techniques can provide positions of terminals with sufficient accuracy for beam pointing and acquisition[17]. In all such cases there has been little work on the engineering and miniaturization required to make these systems robust for widespread deployments.

\subsection{Architectures and operation}

Any communications system must be able to provide service to a number of terminals, and provide both uplink and downlink transmission. Mirror based steering systems are intrinsically point-to-point so in this case the challenge is to provide access to a number of terminals sequentially. SLM based systems can display several steering holograms simultaneously, and therefore provide point-to-multipoint access[18]. Wavelength based steering systems can achieve this by using multiple wavelengths simultaneously.

Providing an uplink (from terminal to base station) is also challenging. For systems with steering at both transmitter and receiver an uplink is often available as a consequence of the symmetry of the system, but other methods exist. As examples, in [19] high-frequency RF is used as the uplink and in [20] the use of remodulated light from the downlink is demonstrated. 


\section{SYSTEM EXAMPLE}

In this section, an SLM based system, developed at Oxford in demonstrated in partnership with researchers at University College London is described, in order to underline the challenges in such approaches. (The results shown here have been reported in a number of papers[15, 21, 22]. Figure 2 shows the system. Light at 1550nm from an optical fibre is collimated and illuminates an SLM that displays a phase-only beamsteering hologram. Light is steered through an angle of up to +/- 3 degrees, depending on the hologram that is displayed, and the angle is then magnified using a $\sim 10 \mathrm{x}$ beamcompressor implemented using commercially available optics. The resulting beam then propagates over several metres to a symmetrical terminal. Aberration correction is implemented by adding additional phase elements to the SLM, and this compensates for the distortions created by the beam magnification system.

Tracking is implemented using two systems. At the base station a CMOS camera is used to image the coverage area, and each terminal has a ring of Infra-red LEDs around the receiver input/output aperture. Matlab is used to process images of the rings acquired using the camera, and this allows position and approximate range to be obtained. At the terminal a beamsplitter is used to 'tap-off' a portion of the incoming light. This illuminates a commercial quad-detector, which allows the angle of arrival of the light to be determined, and thus the required receiver SLM steering angle.

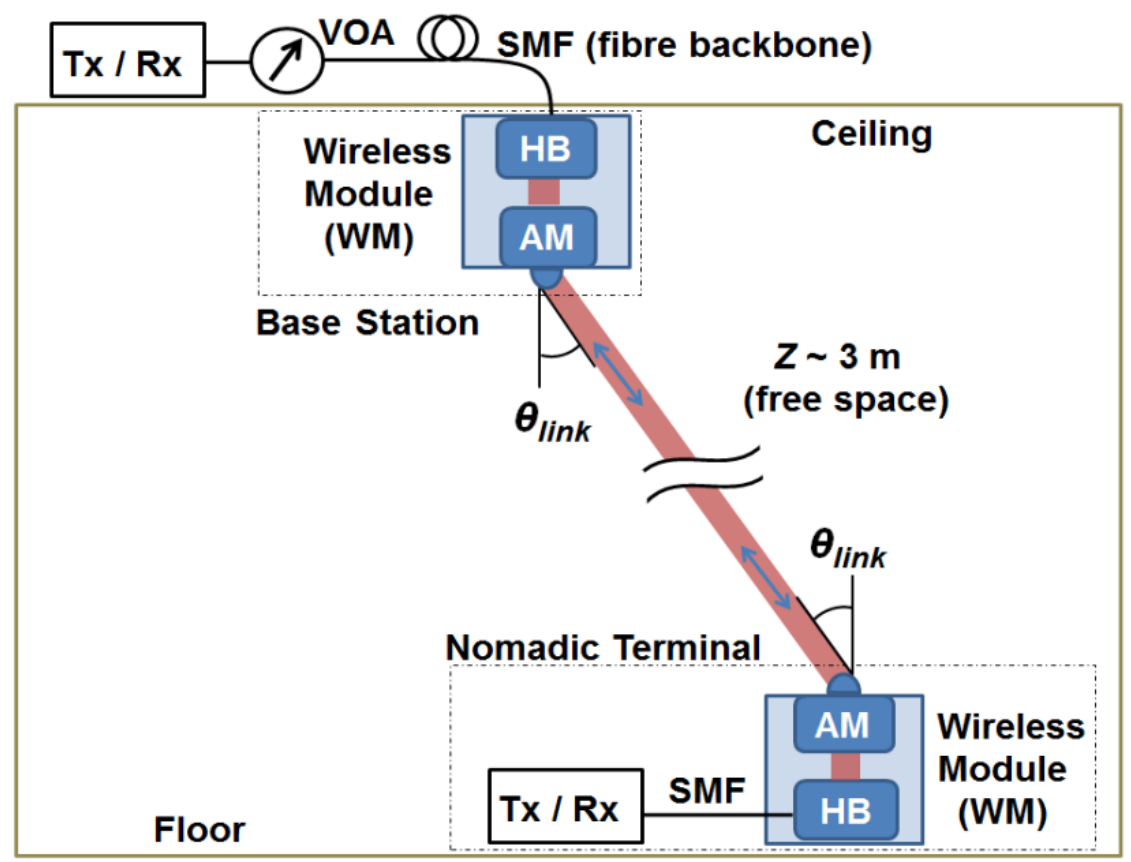

Figure 2. SLM based system. HB-Holographic Beamsteerer. AM-Angle Magnifier

In operation, holograms with aberration correction for each steering angle are used to steer the light from the input fibre towards the receiver at the base station, and the light received back into receiver fibre at the terminal. The holograms at transmitter and receiver are constrained to be identical, so that the system will work symmetrically.

The system was tested using a state-of-the-art fibre transmission system at the UCL networks group in the UK. A coherent Nyquist Wavelength Division Multiplexed (WDM) system was used to transmit up to 14 wavelengths, each containing 30Gbit/s Quadrature Amplitude Modulated (QAM) data (see [15] for a complete description). Figure 3 shows a plot of error rate vs. power margin, where margin is defined as the additional power available for transmission at the error rate shown. Assuming that Forward Error Correction coding (FEC) is available then at the threshold of error correction a data rate of $\sim 420 \mathrm{Gbit} / \mathrm{s}$ was achieved at a steering angle of 20 degrees, in both link directions, and a rate of $\sim 220 \mathrm{Gbit} / \mathrm{s}$ at a steering angle of 30 degrees. The reduction in rate is due to the angular dispersion of the SLM, which reduces the optical bandwidth of the system at higher angles, thus limiting the number of WDM channels that can be transmitted. 


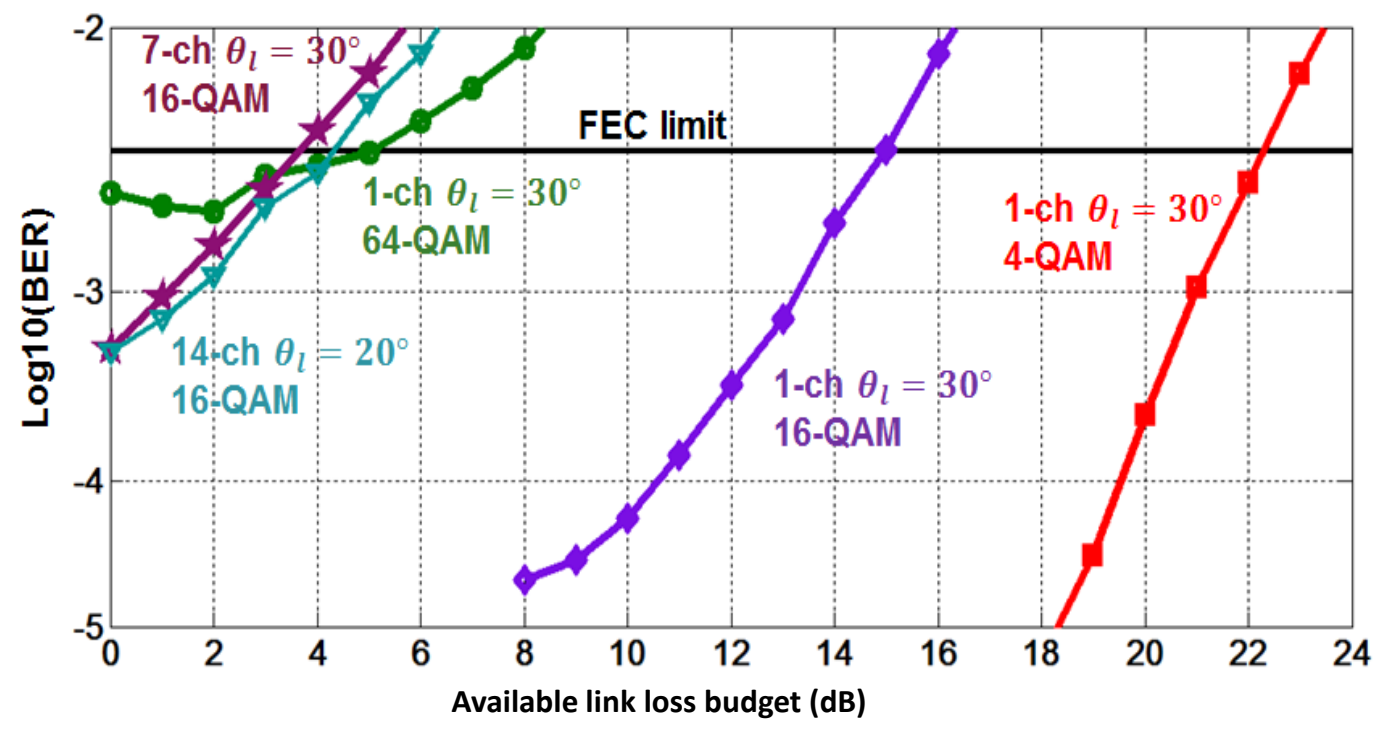

Figure 3. Performance of SLM based beamsteering system.

\section{CONCLUSIONS}

Beamsteering systems will see more widespread use for both RF and Optical wireless in the coming years, as spectrum is exhausted, and link budget limitations make it impossible to achieve the rates required for applications such as virtual reality and wired Ethernet replacement. Beamsteering for optical wireless offers the promise of a system that can be 'transparent' and is directly compatible with the increasingly all-optical fixed communications network. However, there are significant architectural and engineering challenges to create the low-cost compact devices and subsystems required to achieve this.

\section{REFERENCES}

[1] Optotune. (Accessed 2018). www.optotune.com.

[2] Mirrocle. (2018). http://mirrorcletech.com/.

[3] J. Morrison, M. Imboden, T. D. C. Little, and D. J. Bishop, "Electrothermally actuated tip-tilt-piston micromirror with integrated varifocal capability," Optics Express, vol. 23, pp. 9555-9566, Apr 62015.

[4] N. R. Smith, D. C. Abeysinghe, J. W. Haus, and J. Heikenfeld, "Agile wide-angle beam steering with electrowetting microprisms," Opt. Express, vol. 14, pp. 6557-6563, 2006.

[5] D. Kopp, L. Lehmann, and H. Zappe, "Optofluidic laser scanner based on a rotating liquid prism," Applied Optics, vol. 55, pp. 2136-2142, Mar 202016.

[6] F. Feng, I. H. White, and T. D. Wilkinson, "Holographic beam steering a directly modulated two-electrode high brightness tapered laser diode for optical wireless communications," 2012 Asia Communications and Photonics Conference (Acp), 2012.

[7] D. C. O'Brien, G. E. Faulkner, B. Robertson, and T. D. Wilkinson, "Adaptive board-to-board optical interconnects that use dynamic holograms," in IOP topical meeting on SLMs and applications, London, 2003.

[8] D. Engstrom, S. Hard, P. Rudquist, K. D'Have, T. Matuszczyk, M. Skeren, and B. Lofving, "Beam steering by combing two binary-phase-modulated FLC SLMs," Proceedings of the SPIE The International Society for Optical Engineering, vol. 5181, pp. 132-43, 2003.

[9] J. C. Hulme, J. K. Doylend, M. J. R. Heck, J. D. Peters, M. L. Davenport, J. T. Bovington, L. A. Coldren, and J. E. Bowers, "Fully integrated hybrid silicon two dimensional beam scanner," Optics Express, vol. 23, pp. 58615874, Mar 92015. 
[10] K. Van Acoleyen, W. Bogaerts, J. Jágerská, N. Le Thomas, R. Houdré, and R. Baets, "Off-chip beam steering with a one-dimensional optical phased array on silicon-on-insulator," Optics Letters, vol. 34, pp. 1477-1479, 2009/05/01 2009.

[11] A. Yaacobi, J. Sun, M. Moresco, G. Leake, D. Coolbaugh, and M. R. Watts, "Integrated phased array for wideangle beam steering," Optics Letters, vol. 39, pp. 4575-4578, 2014/08/01 2014.

[12] C. W. Oh, Z. Z. Cao, E. Tangdiongga, and T. Koonen, "Free-space transmission with passive 2D beam steering for multi-gigabit-per-second per-beam indoor optical wireless networks," Optics Express, vol. 24, pp. 1921119227, Aug 222016.

[13] K. Liang, H. Shi, S. J. Sheard, and D. C. O'Brien, "Transparent Optical Wireless Hubs Using Wavelength Space Division Multiplexing," in Free-Space Laser Communications IV, Denver, 2004, pp. 80-87.

[14] K. Wang, A. Nirmalathas, C. Lim, and E. Skafidas, "High-Speed Indoor Optical Wireless Communication System with a Steering Mirror Based Up-Link Receiver," 2012 38th European Conference and Exhibition on Optical Communications (Ecoc), 2012.

[15] A. Gomez, K. Shi, C. Quintana, R. Maher, G. Faulkner, P. Bayvel, B. C. Thomsen, and D. O'Brien, "Design and Demonstration of a $400 \mathrm{~Gb} / \mathrm{s}$ Indoor Optical Wireless Communications Link," Journal of Lightwave Technology, vol. 34, pp. 5332-5339, Nov 152016.

[16] A. Gomez, K. Shi, C. Quintana, G. Faulkner, B. C. Thomsen, and D. O’Brien, "A 50 Gbit/s Transparent Indoor Optical Wireless Communications Link With an Integrated Localization and Tracking System," Journal of Lightwave Technology, vol. 34, pp. 2510-2517, 2016.

[17] K. Wang, A. Nirmalathas, C. Lim, and E. Skafidas, "High-speed duplex optical wireless communication system for indoor personal area networks," Optics Express, vol. 18, pp. 25199-25216, Nov 222010.

[18] Vachiramon-P, Faulkner-Ge, and Obrien-Dc, "A DC balancing algorithm for FLCOS binary phase holograms," Optics Letters, vol. 32, pp. 3275-7, 15 Nov. 2007.

[19] A. M. Khalid, P. Baltus, A. R. Dommele, K. A. Mekonnen, Z. Cao, C. W. Oh, M. K. Matters, and A. M. J. Koonen, "Bi-directional 35-Gbit/s 2D Beam Steered Optical Wireless Downlink and 5-Gbit/s Localized 60GHz Communication Uplink for Hybrid Indoor Wireless Systems," 2017 Optical Fiber Communications Conference and Exhibition $(O f c), 2017$.

[20] C. W. Oh, Z. Cao, K. A. Mekonnen, E. Tangdiongga, and A. M. J. Koonen, "Low-Crosstalk Full-Duplex AllOptical Indoor Wireless Transmission With Carrier Recovery," Ieee Photonics Technology Letters, vol. 29, pp. 539-542, Mar 152017.

[21] A. Gomez, K. Shi, C. Quintana, M. Sato, G. Faulkner, B. C. Thomsen, and D. O'Brien, "Beyond 100-Gb/s Indoor Wide Field-of-View Optical Wireless Communications," Ieee Photonics Technology Letters, vol. 27, pp. 367-370, Feb 152015.

[22] A. Gomez, C. Quintana, G. Faulkner, and D. O'Brien, "Challenges in Wide Coverage Indoor Optical Communications Using Fibre-Wireless-Fibre Links for Terabit data rates," 2015 Ieee Globecom Workshops (Gc Wkshps), 2015. 